\title{
Cholesterol esterification enzymes promote cancer growth and are potential therapeutic targets for repurposed drugs: a systematic review and meta-analysis of pre-clinical evidence
}

\author{
A. Websdale ${ }^{1}$, P. Chalmers ${ }^{1}$, X. Chen ${ }^{1}$, Y. Kiew ${ }^{1}$, X. Luo ${ }^{1}$, R. Mwarazi ${ }^{1}$, R. Wu ${ }^{1}$, \\ G. Cioccoloni ${ }^{1}$, H. Røberg-Larsen ${ }^{2}$, T.A. Hughes ${ }^{3}$, M.A. Zulyniak ${ }^{1}$ and J.L. Thorne ${ }^{1}$ \\ ${ }^{1}$ School of Food Science and Nutrition, University of Leeds, Leeds, UK, \\ ${ }^{2}$ Department of Chemistry, University of Oslo, Norway and School of Medicine, Norway and \\ ${ }^{3}$ School of Medicine, University of Leeds, Leeds, UK
}

Cholesterol esterification enzymes sterol-O acyltransferases 1 and 2 (SOAT) have been reported as prognostic markers in several cancers $^{(1,2)}$. These enzymes utilise fatty acids conjugated to coenzyme A to generate ester groups at C3 of cholesterol. Cholesterol esterification enables the formation of lipid droplets that act as storage organelles for lipid soluble vitamins and minerals, and as cholesterol reservoirs. There is a high demand for rapid access to cholesterol during tumour growth to maintain continual synthesis of the plasma membrane for which cholesterol is indispensable. Anti-cancer therapies that block cholesterol storage are underexplored in the clinical setting, yet a plethora of in vitro and preclinical data suggest the time is right to target this pathway in clinical trials. In this systematic review and meta-analysis, we summarise the current state of understanding of this metabolic pathway in pancancer development and evaluate current evidence of the molecular pathways altered by interventions that target cholesterol esterification.

We performed a systematic search of PubMed, Scopus, and Web of Science to identify preclinical studies that had evaluated differences in cholesterol ester concentration in tumour and non-tumour tissue, and the effect of inhibiting esterification on tumour growth (PROSPERO: CRD42020202409). After deduplication, 1482 abstracts were screened for eligibility by two independent researchers, leading to full text screening of 59 records. Eight studies evaluating cholesterol ester concentration between tumour and matched nontumour tissue and 21 studies evaluating genetic or pharmacological inhibition of esterification were included for data extraction. Mean difference (MD) was used where possible for reporting changes to ester concentrations or tumour size, standardised mean difference (SMD) was used otherwise; both were reported with random effects modelling.

Tumour tissue had a significantly greater concentration of cholesterol esters than non-tumour tissue $(\mathrm{SMD}=1.1 ; \mathrm{p}<0.0001)$. Pharmacological (Avasimibe, ATR-101, K-604) or genetic inhibition (siRNA, Cre/flox) of SOAT1/2 was associated with significantly smaller tumours of the brain $(\mathrm{SMD}=-3.28 ; \mathrm{p}<0.00001)$, liver $(\mathrm{MD}=-0.28 \mathrm{~cm} 3 ; \mathrm{p}<0.002)$, pancreas $(\mathrm{MD}=-0.56 \mathrm{~cm} 3 ; \mathrm{p}<0.0001)$, prostate $(\mathrm{SMD}=-1.64 ; \mathrm{p}=0.004)$, and skin $(\mathrm{SMD}=-3.44 ; \mathrm{p}<0.00001)$. Additionally, reduced tumour volume was accompanied with increased apoptosis $(\mathrm{SMD}=5.64, \mathrm{p}=0.0004), \mathrm{CD} 8+$ immune infiltration $(\mathrm{SMD}=1.12, \mathrm{p}=0.0009)$ and reduced Ki67 expression $(\mathrm{SMD}=-3.27, \mathrm{p}=0.0001)$. We noted a significant risk of publication bias; trim and fill method indicated our observed effect size may be an overestimate by as much as $28 \%$.

Avasimibe was the most commonly used pharmacological SOAT inhibitor. Repurposing of Avasimibe and other SOAT inhibitors appears plausible as the anti-cancer properties we summarise here occur at doses equivalent to those previously reported safe and tolerable in clinical trials for treatment of non-oncological diseases ${ }^{(3,4)}$. This work indicates that SOAT1/2 inhibition should be explored in clinical trials as an adjunct to existing anti-neoplastic agents.

\section{References}

1. Chi K-C, et al. (2019) Mol Neurobiol, 56.

2. Jiang Y, et al. (2019) Nature, $\mathbf{5 6 7}$

3. Raal FJ, et al. (2003) Atherosclerosis, 171.

4. Tardif J-C, et al. (2004) Circulation, 110. 